\title{
Applying the WHO ICF Framework to the Outcome Measures Used in the Evaluation of Long-Term Clinical Outcomes in Coronavirus Outbreaks
}

\author{
Kajal Patel ${ }^{1,2}\left(\mathbb{D}\right.$, , Sofia Straudi ${ }^{3}, \mathrm{Ng}$ Yee Sien ${ }^{4}$, Nora Fayed ${ }^{5}$, John L. Melvin ${ }^{6}{ }^{\oplus}$ and \\ Manoj Sivan $2,7, *$ (D) \\ 1 School of Medicine, University of Manchester, Manchester M13 9PG, UK; kj.patel1020@gmail.com \\ 2 Division of Neurosciences and Experimental Psychology, University of Manchester, \\ Manchester M13 9PL, UK \\ 3 Neuroscience and Rehabilitation Department, Ferrara University Hospital, 44100 Ferrara, Italy; \\ s.straudi@ospfe.it \\ 4 Department of Rehabilitation Medicine, Singapore General Hospital, Outram Road, \\ Singapore 169608, Singapore; ng.yee.sien@singhealth.com.sg \\ 5 School of Rehabilitation Therapy, Queen's University, Louise D. Acton Building, 31 George Street, \\ Kingston, ON K7L 3N6, Canada; nf31@queensu.ca \\ 6 Department of Rehabilitation Medicine, Sidney Kimmel Medical College, Thomas Jefferson University, \\ Philadelphia, PA 19144, USA; honpres@hotmail.com \\ 7 Academic Department of Rehabilitation Medicine, University of Leeds and Leeds Teaching Hospitals NHS \\ Trust, Leeds LS2 9JT, UK \\ * Correspondence: m.sivan@leeds.ac.uk
}

Received: 3 August 2020; Accepted: 1 September 2020; Published: 5 September 2020

Abstract: (1) Objective: The World Health Organization's (WHO) International Classification of Functioning, Disability and Health (ICF) classification is a unified framework for the description of health and health-related states. This study aimed to use the ICF framework to classify outcome measures used in follow-up studies of coronavirus outbreaks and make recommendations for future studies. (2) Methods: EMBASE, MEDLINE, CINAHL and PsycINFO were systematically searched for original studies assessing clinical outcomes in adult survivors of severe acute respiratory distress syndrome (SARS), middle east respiratory syndrome (MERS) and coronavirus disease-19 (COVID-19) after hospital discharge. Individual items of the identified outcome measures were linked to ICF second-level and third-level categories using ICF linking rules and categorized according to an ICF component. (3) Results: In total, 33 outcome measures were identified from 36 studies. Commonly used (a) ICF body function measures were Pulmonary Function Tests (PFT), Impact of event scale (IES-R) and Hospital Anxiety and Depression Scale (HADS); (b) ICF activity was 6-Minute Walking Distance (6MWD); (c) ICF participation measures included Short Form-36 (SF-36) and St George's Respiratory Questionnaire (SGRQ). ICF environmental factors and personal factors were rarely measured. (4) Conclusions. We recommend future COVID-19 follow-up studies to use the ICF framework to select a combination of outcome measures that capture all the components for a better understanding of the impact on survivors and planning interventions to maximize functional return.

Keywords: COVID-19; SARS; MERS; outcome measures; follow-up studies; prevalence; lung function; exercise tolerance; mental health; quality of life

\section{Introduction}

Coronavirus disease 2019 (COVID-19) has posed a major challenge to healthcare systems worldwide, with over 6 million confirmed cases and 350,000 deaths reported as of May 2020 [1]. 
Whilst current efforts have been duly focused on reducing mortality, issues regarding potential long-term complications in COVID-19 survivors are starting to emerge [2-4]. In the previous coronavirus outbreaks of severe acute respiratory syndrome (SARS) and Middle East respiratory syndrome (MERS), such long-term sequelae spanned across the physical, psychological and social domains of health [5,6]. Early reports of COVID-19 follow-up studies are suggestive of prevalence of similar health problems in COVID-19 survivors as those reported in previous outbreaks of SARS and MERS [7,8]. Considering the multi-systemic impact of COVID-19, follow-up studies across all these domains of health are required in order to truly understand these individuals' experiences and support their return to societal roles.

Post-acute early rehabilitation of coronavirus survivors would foremost involve recovery of body structure and function. Impairment of respiratory function [9-11], exercise tolerance [12] and neuromuscular functions [13] have been reported to be present in coronavirus survivors beyond 3 months post-infection, with improvement to some degree in many of these individuals. However, improvement in these impairments of body structure and function may not necessarily translate into recovery from disability and role limitation $[14,15]$. For instance, during the SARS outbreak in 2002, even though most patients had good recovery from their physical illness, their quality of life was still lower than healthy individuals and as many as $17 \%$ of coronavirus survivors had not returned to work 1-year post-discharge [11]. Furthermore, evaluation of impairment without the knowledge of personal factors and help available to them in their environments can be futile as barriers in these contextual factors need to be overcome to facilitate participation of the individual in society.

In order to assess survivors' long-term health comprehensively and provide rehabilitation, all aspects of health that influence recovery, including organ impairments, functional limitations and personal circumstances, should be assessed. The World Health Organization's International Classification of Functioning, Disability and Health (WHO ICF) model put forward in 2001 provides a coherent view of different aspects of health from biological, individual and social perspectives [16,17]. This framework has been extensively researched and validated for describing health state [17], epidemiology [18] and public health [18], classifying outcome measures [15,19] and planning interventions [16,20]. The interplay between these factors in COVID-19 survivors is important to recognize in order to characterize their multi-systemic problems and disability and improve "functioning" by targeted interventions.

The aim of this systematic review is to identify outcome measures which have been used in follow-up studies in the coronavirus outbreaks, including SARS in 2002 and MERS in 2012 [21], and to classify them using the ICF model. This will allow an understanding of whether all aspects of the health condition have been captured by these studies and highlight any gaps that need to be considered when selecting outcome measures for future studies.

\section{Methods}

This systematic review was conducted in two stages.

\subsection{Stage 1. Identification of Long-Term Follow-Up Studies in Survivors of Previous Coronavirus Outbreaks}

A comprehensive search of 4 databases, MEDLINE (1946 to Week 1 May 2020), EMBASE (1974 to 8th May 2020), CINAHL Plus (1937 to Week 1 May 2020) and PsycINFO (1806 to Week 1 May 2020), was performed. The search strategy used was [(Coronavirus OR Coronavirus Infections OR COVID OR SARS virus OR Severe acute respiratory syndrome OR MERS OR Middle east respiratory syndrome) AND (Follow-up OR Follow-up studies OR Prevalence)]. Terms were entered as MeSH terms where available for each database; otherwise, these were searched as keywords in the title, abstract and subject headings.

The searches were first screened using the abstract and then using the full text by two independent authors based on the inclusion and exclusion criteria below. Original studies involving adults with confirmed diagnosis of coronavirus infection who were followed up for any period post-discharge 
were included. Further inclusion was limited to studies that used at least one clinical outcome measure during the follow-up (e.g., studies investigating mortality only were excluded). Reviews, case reports and editorial reports were excluded. Information regarding the authors, study year and outcome measures used in all the included studies was extracted into standardized tables. Extraction was undertaken by KP and MS independently and then compared for accuracy.

\subsection{Stage 2. Classification of Outcome Measures According to the Five Main ICF Components}

ICF linking rules were used in this study. These rules have been developed specifically to link the content of each outcome measure to the ICF framework [22]. The rules suggest that meaningful concepts within the items of outcome measures should be identified and then linked to the most precise ICF category. Meaningful "concepts" are those that describe health condition, person, functional activity or any of the environmental factors. For example, the measure IES-R scale (Impact of Event Scale-Revised) has two items, "I had trouble staying asleep" and "I felt angry", the concepts extracted from these items were sleep and anger. Sleep was linked to the second level ICF category, "b134-sleep function", and anger was linked to the third level ICF category, "b1522-anger". Meaningful concepts referring to "quality of life" are assigned to the "not definable-quality of life" category. If a meaningful concept is not contained in the ICF and is clearly a personal factor, it is assigned "personal factor". If a meaningful concept is not contained in ICF and is not a personal factor, it is assigned "not covered". If the meaningful concept refers to a diagnosis or a health condition, it is assigned "health condition" [22]. Based on the above rules, meaningful concepts were extracted from the items of the identified measures and linked to relevant ICF categories. Finally, each outcome measure was then classified to belong to the ICF component that covered the majority of its constituent questions. For example, the items of GHQ-12 were linked to the two ICF components, "body function and structures" and "activities", with the majority of questions linking to the ICF "body function and structures" component. Hence, this outcome measure was classified as belonging to (or representing) ICF "body function and structures". This stage was undertaken by authors KP and MS and cross-checked for consensus by NYS and NF.

Based on the spread of the these meaningful concepts, each outcome measure was classified into one of the main individual ICF components [22], defined as follows:

- Body structure and body function: refers to anatomical structure or physiological function such as those required for cognition, cardiovascular function, motor functions, pain or emotion.

- Activities: refers to the execution of tasks at an individual level.

- Participation: refers to the individual's involvement in everyday life situations.

- Environmental factors: refer to physical, social and attitudinal factors in the person's life and society which hinder or facilitate the functioning of the individual.

- Personal factors: refers to characteristics that are unique to each individual such as age, gender, ethnicity, personality, resilience or experiences.

This stage was undertaken independently by all authors and cross-checked for consensus.

\section{Results}

\subsection{Stage 1. Study Selection}

The search of four databases yielded 1528 studies. Out of these, 36 studies were finally included in this review. The reasons for exclusion of studies at each stage are reported in Figure 1. 


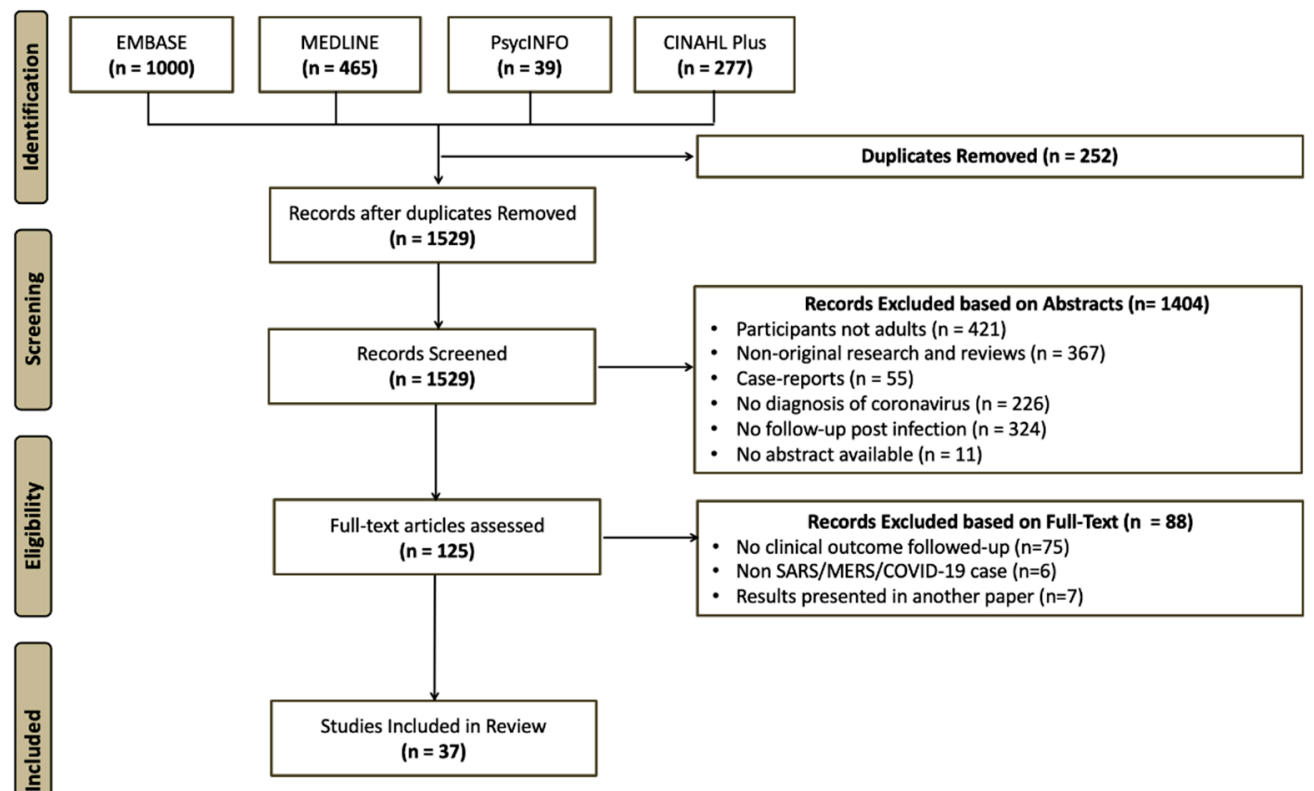

Figure 1. PRISMA flowchart for the literature search.

\subsection{Stage 2. ICF Outcome Measures}

Tables 1 and 2 summarizes the outcome measures used by the follow-up studies in coronavirus survivors included in this review. A total of 33 outcome measures were identified which measured several physical, psychological and quality of life outcomes. The commonly used outcome measures included Pulmonary Function Tests (PFT; 20 studies), Impact of Event Scale (IES-R; seven studies), Short-Form 36 (SF-36; six studies), 6-Minute Walking Distance (6MWD; five studies), Hospital Anxiety and Depression Scale (HADS; three studies) and St George's Respiratory Questionnaire (SQRG; three studies).

The mapping of meaningful concepts to the ICF categories is shown in Table 3. Based on how the units mapped, the authors decided on which ICF component each outcome measure ultimately represents. Figure 2 portrays the classification of outcome measures based on ICF components. In total, 19 outcome measures measured ICF body structure and function, three outcome measures measured activity, four outcome measures measured participation, one outcome measure measured environmental factors and seven outcome measures measured personal factors (see Table 1).

Table 1. Outcome measure abbreviations.

\begin{tabular}{cc}
\hline Abbreviation & Outcome Measure \\
\hline PFT & Pulmonary Function Test \\
IES-R & Impact of Event Scale-Revised \\
HADS & Hospital Anxiety and Depression Scale \\
SCID & Structures Clinical Interview for DSM Disorders \\
SDS & Self-rating Depression Scale \\
SAS & Self-rating Anxiety Scale \\
SCL-90 & Symptom Checklist 90 \\
PSS-10 & Perceived Stress Scale \\
GHQ-12 & General Health Questionnaire 12 \\
DASS & Depression Anxiety Stress Scale \\
PHQ & Patient Health Questionnaire 9 \\
PTSD-SS & PTSD Self-rating Scale \\
CFS & Chalder Fatigue Scale \\
FSS & Fatigue Severity Scale \\
\hline
\end{tabular}


Table 1. Cont.

\begin{tabular}{cc}
\hline Abbreviation & Outcome Measure \\
\hline MRC Muscle Power & Medical Research Council Muscle Power \\
NMS Exam & Neuromuscular Examination \\
6MWD & 6-Minute Walking Distance \\
CPET & Cardiopulmonary Exercise Testing \\
FIM & Functional Independence Measure \\
SGRQ & St George's Respiratory Questionnaire \\
FIC & Functional Impairment Checklist \\
SDSS & Social Disability Screening Schedule \\
SF-36 & Short-Form 36 \\
PSSS & Perceived Social Support Scale \\
SCSQ & Simple Coping Style Questionnaire \\
SES & Self-Esteem Scale \\
EPQ & Eysenck Personality Questionnaire \\
\hline
\end{tabular}

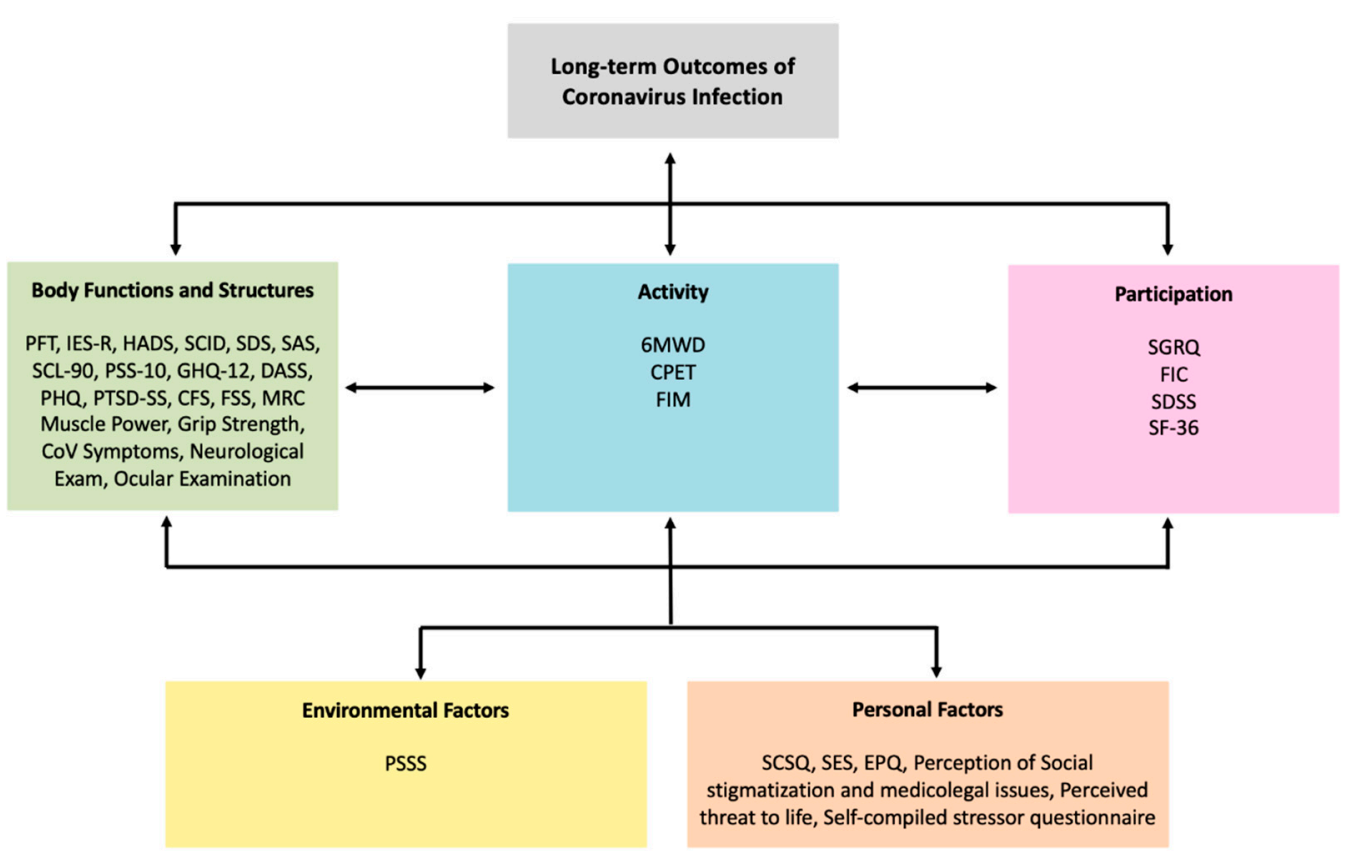

Figure 2. International Classification of Functioning, Disability and Health (ICF) categorization of outcome measures used in long-term follow-up studies of coronavirus survivors. 
Table 2. Outcome measures used in follow-up studies of coronavirus survivors.

\begin{tabular}{|c|c|c|c|c|c|c|c|c|c|c|c|c|c|c|c|c|c|c|c|c|c|c|c|c|c|c|c|c|c|c|c|}
\hline \multirow[b]{2}{*}{ 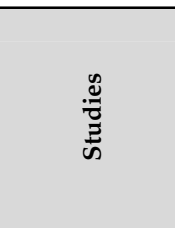 } & \multicolumn{31}{|c|}{ Outcome Measures Used in Follow-Up Studies of Coronavirus Survivors } \\
\hline & 氞 & 总 & 空 & 己े & की & क्ष & ì & 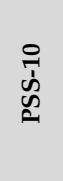 & 竎 & $\sum_{0}^{n}$ & $\underset{\hat{d}}{\hat{d}}$ & 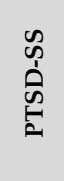 & 侃 & w & 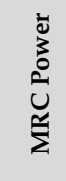 & 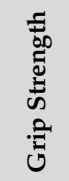 & $\begin{array}{l}\text { है } \\
\text { है } \\
\text { हूँ } \\
\text { के }\end{array}$ & 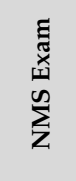 & 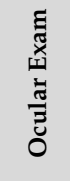 & $\sum_{b}^{0}$ & Ũ & $\sum_{i=1}$ & $\begin{array}{l}\infty \\
\frac{1}{\omega} \\
\frac{1}{\omega}\end{array}$ & \begin{tabular}{l} 
a \\
\multirow{J}{W}{} \\
\end{tabular} & $\underline{U}$ & के & W & O్ & 急 & $\underset{t}{O}$ & 异 \\
\hline $\begin{array}{l}\text { Mak et al. } \\
\text { (2009) [23] }\end{array}$ & & + & + & + & & & & & & & & & & & & & & & & & & & + & & & & & & & & \\
\hline $\begin{array}{l}\text { Lam et al. } \\
\text { (2009) [24] }\end{array}$ & & + & + & + & & & & & & & & & + & & & & & & & & & & & & & & & & & & $\begin{array}{l}\text { Perception of social } \\
\text { stigmatization and } \\
\text { medicolegal issues }\end{array}$ \\
\hline $\begin{array}{l}\text { Wu et al. } \\
\text { (2005) [25] }\end{array}$ & & + & + & & & & & & & & & & & & & & & & & & & & & & & & & & & & $\begin{array}{l}\text { 5-point scale measuring } \\
\text { perceived threat to life }\end{array}$ \\
\hline $\begin{array}{l}\text { Lee et al. } \\
\text { (2007) [26] }\end{array}$ & & + & & & & & & + & + & + & & & & & & & & & & & & & & & & & & & & & \\
\hline $\begin{array}{l}\text { Lee et al. } \\
\text { (2019) [27] }\end{array}$ & & + & & & & & & & & & + & & & + & & & & & & & & & & & & & & & & & \\
\hline $\begin{array}{l}\text { Zhang et al. } \\
\text { (2005) [28] }\end{array}$ & & + & & & & & & & & & & & & & & & & & & & & & & & & & + & + & + & + & $\begin{array}{l}\text { Self-compiled stressor } \\
\text { questionnaire }\end{array}$ \\
\hline $\begin{array}{l}\text { Hong et al. } \\
\text { (2009) [29] }\end{array}$ & & + & & & + & + & + & & & & & & & & & & & & & & & & + & & & + & & & & & \\
\hline $\begin{array}{l}\text { Liu et al. } \\
\text { (2020) [30] }\end{array}$ & + & & & & + & + & & & & & & & & & & & & & & + & & + & + & & & & & & & & \\
\hline $\begin{array}{l}\text { Lam et al. } \\
\text { (2006) [31] }\end{array}$ & & & & & & & & & & & & & & & & + & & & & + & & & + & + & + & & & & & & \\
\hline $\begin{array}{l}\text { Peng et al. } \\
\text { (2003) [18] }\end{array}$ & + & & & & & & & & & & & & & & & & & & & & & & & & & & & & & & \\
\hline $\begin{array}{l}\text { Xie et al. } \\
\text { (2005) [32] }\end{array}$ & + & & & & & & & & & & & & & & & & & & & & & & & & & & & & & & \\
\hline $\begin{array}{l}\text { HE et al. } \\
\text { (2005) [33] }\end{array}$ & + & & & & & & & & & & & & & & & & & & & & & & & & & & & & & & \\
\hline $\begin{array}{l}\text { Ong et al. } \\
\text { (2004) [34] }\end{array}$ & + & & & & & & & & & & & & & & & & & & & & + & & & & & & & & & & \\
\hline $\begin{array}{l}\text { Ng et al. } \\
\text { (2005) [35] }\end{array}$ & + & & & & & & & & & & & & & & & & & & & & & & & & & & & & & & \\
\hline $\begin{array}{l}\text { Zheng et al. } \\
\text { (2005) [36] }\end{array}$ & + & & & & & & & & & & & & & & & & & & & & & & & & & & & & & & \\
\hline $\begin{array}{l}\text { Hsu et al. } \\
\text { (2004) [37] }\end{array}$ & + & & & & & & & & & & & & & & & & + & & & & & & & & & & & & & & \\
\hline $\begin{array}{l}\text { Gao et al. } \\
\text { (2006) [38] }\end{array}$ & & & & & & & & & & & & + & & & & & & & & & & & & & & & & & & & \\
\hline $\begin{array}{l}\text { Zheng-Yu et } \\
\text { al. (2003) [39] }\end{array}$ & + & & & & & & & & & & & & & & & & & & & & & & & & & & & & & & \\
\hline
\end{tabular}


Table 2. Cont.

\begin{tabular}{|c|c|c|c|c|c|c|c|c|c|c|c|c|c|c|c|c|c|c|c|c|c|c|c|c|c|c|c|c|c|c|c|}
\hline \multirow[b]{2}{*}{ 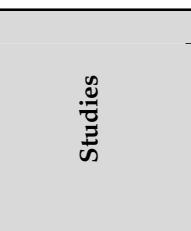 } & \multicolumn{31}{|c|}{ Outcome Measures Used in Follow-Up Studies of Coronavirus Survivors } \\
\hline & 点 & 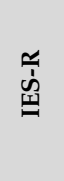 & 空 & : & के & के & ষ্ণ & $\begin{array}{l}\text { 定 } \\
\text { के } \\
\text { a }\end{array}$ & ำ & 点 & 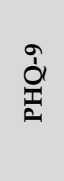 & $\begin{array}{l}\infty \\
\omega \\
\omega \\
\omega\end{array}$ & 侃 & क् & 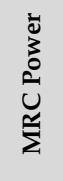 & 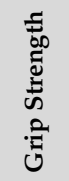 & $\begin{array}{l}\text { है } \\
\text { है } \\
\text { है } \\
\text { के }\end{array}$ & 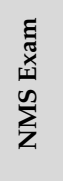 & 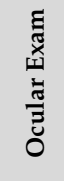 & $\sum_{0}^{0}$ & 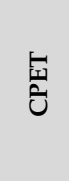 & $\sum_{\text {II }}$ & $\begin{array}{l}\infty \\
\frac{1}{\omega}\end{array}$ & $\begin{array}{l}0 \\
\text { U্ } \\
\infty\end{array}$ & U & की & W & O্ & 兽 & Oa & ț \\
\hline $\begin{array}{l}\text { Yun et al. } \\
\text { (2003) [40] }\end{array}$ & + & & & & & & & & & & & & & & & & & & & & & & & & & & & & & & \\
\hline $\begin{array}{l}\text { Liu et al. } \\
\text { (2007) [41] }\end{array}$ & + & & & & & & & & & & & & & & & & & & & & & & & & & & & & & & \\
\hline $\begin{array}{l}\text { Ong et al. } \\
\text { (2005) [42] }\end{array}$ & + & & & & & & & & & & & & & & & & & & & & & & & + & & & & & & & \\
\hline $\begin{array}{l}\text { Zhang et al. } \\
\text { (2020) [43] }\end{array}$ & + & & & & & & & & & & & & & & & & & & & & & & & & & & & & & & \\
\hline $\begin{array}{l}\text { Tsai et al. } \\
\text { (2004) [13] }\end{array}$ & & & & & & & & & & & & & & & + & & & & + & & & & & & & & & & & & \\
\hline $\begin{array}{l}\text { Chen et al. } \\
\text { (2006) [10] }\end{array}$ & & & & & & & & & & & & & & & & & + & & & & & & & & & & & & & & \\
\hline $\begin{array}{l}\text { Hui et al. } \\
\text { (2005) [9] }\end{array}$ & + & & & & & & & & & & & & & & & & & & & + & & & + & & & & & & & & \\
\hline $\begin{array}{l}\text { Wong et al. } \\
\text { (2004) [44] }\end{array}$ & + & & & & & & & & & & & & & & & & & & & & & & & & & & & & & & \\
\hline $\begin{array}{l}\text { Li et al. (2006) } \\
\text { [45] }\end{array}$ & + & & & & & & & & & & & & & & & & & & & + & & & + & & & & & & & & \\
\hline $\begin{array}{l}\text { Park et al. } \\
\text { (2018) [12] }\end{array}$ & + & & & & & & & & & & & & & & & & & & & + & & & & & & & & & & & \\
\hline $\begin{array}{l}\text { Chiang et al. } \\
\text { (2004) [46] }\end{array}$ & + & & & & & & & & & & & & & & & & & & & & & & & & & & & & & & \\
\hline $\begin{array}{l}\text { Yin et al. } \\
\text { (2005) [47] }\end{array}$ & + & & & & & & & & & & & & & & & & & & & & & & & & & & & & & & \\
\hline $\begin{array}{l}\text { Wu et al. } \\
\text { (2016) [48] }\end{array}$ & + & & & & & & & & & & & & & & & & & & & & & & & & & & & & & & \\
\hline $\begin{array}{l}\text { Tansey et al. } \\
\text { (2007) [11] }\end{array}$ & + & & & & & & & & & & & & & & & & + & & & + & & & & + & & & & & & & \\
\hline $\begin{array}{l}\text { Avendano et } \\
\text { al. (2003) [49] }\end{array}$ & & & & & & & & & & & & & & & & & + & & & & & & & & & & & & & & \\
\hline $\begin{array}{l}\text { Isakbaeva et } \\
\text { al. (2004) [50] }\end{array}$ & & & & & & & & & & & & & & & & & + & & & & & & & & & & & & & & \\
\hline $\begin{array}{l}\text { Klopfenstein } \\
\text { et al. (2020) } \\
\text { [51] }\end{array}$ & & & & & & & & & & & & & & & & & + & & & & & & & & & & & & & & \\
\hline $\begin{array}{l}\text { Hopkins et al. } \\
\text { (2020) [52] }\end{array}$ & & & & & & & & & & & & & & & & & + & & & & & & & & & & & & & & \\
\hline $\begin{array}{l}\text { Yuen et al. } \\
\text { (2004) [53] }\end{array}$ & & & & & & & & & & & & & & & & & & + & & & & & & & & & & & & & \\
\hline
\end{tabular}

"+" means that this outcome measure was investigated by the respective study. 
Table 3. Mapping of outcome measure themes to ICF category codes

\begin{tabular}{|c|c|c|c|c|c|c|c|c|c|c|c|c|c|c|c|c|c|c|c|c|c|c|c|c|c|c|c|c|}
\hline & & & & & & & & & & 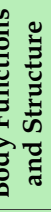 & & & & & & & & & & & 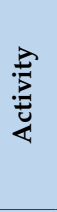 & & & 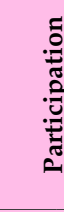 & & & 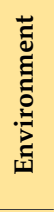 & 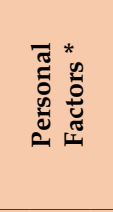 \\
\hline 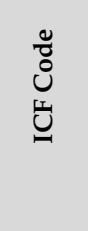 & 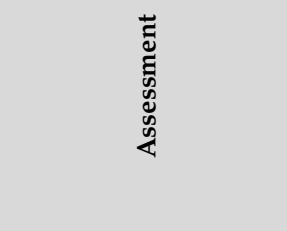 & 至 & 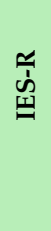 & 空 & 论 & के & $\sum_{\infty}^{\infty}$ & $\mathrm{SCl}$ 离 & GH & 点 & $\stackrel{a}{a}$ & 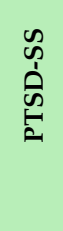 & 品 & w & 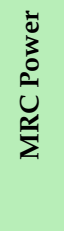 & 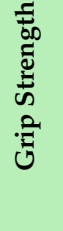 & $\begin{array}{l}\text { है } \\
\text { है } \\
\text { है } \\
\text { के }\end{array}$ & 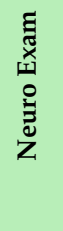 & 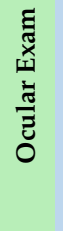 & $\sum_{0}^{\infty}$ & 预 & $\sum_{I}$ & $\begin{array}{l}0 \\
\frac{1}{0} \\
\text { d }\end{array}$ & $\underline{U}$ & के & $\begin{array}{l}\infty \\
\frac{1}{\omega} \\
\frac{1}{\omega}\end{array}$ & के & 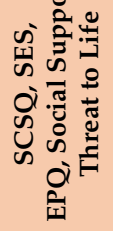 \\
\hline b1263 & Psychic stability & & + & & & & & & & & & & & & & & & & & & & & & & & & & \\
\hline b1266 & Confidence & & & & & & & & + & & & & & & & & & & & & & & & & & & & \\
\hline b130 & $\begin{array}{l}\text { Energy and drive } \\
\text { functions }\end{array}$ & & & & & & & & & & + & & + & + & & & & & & & & & & & & + & & \\
\hline b1302 & Appetite & & & & & & & & & & + & & & & & & & & & & & & & & & & & \\
\hline b134 & Sleep function & & + & & & & & & + & & + & & & & & & & & & & & & + & & & & & \\
\hline b144 & Memory function & & & & & & & & & & & & & & & & & & & & & & & & & & & \\
\hline b152 & Stress & & & & & & & + & & + & & & & & & & & & & & & + & & & & & & \\
\hline b152 & Anxiety & & + & + & & & + & + & & + & & + & & & & & & & & & & & & & & & & \\
\hline b152 & Depression & & & + & + & + & & + & + & + & + & & & & & & & & & & & & & & & + & & \\
\hline b152 & Panic & & & & & & & & & & & & & & & & & & & & & & + & & & & & \\
\hline b152 & Fear & & & + & & & & & & & & & & & & & & & & & & & + & & & & & \\
\hline b152 & Concentrate & & + & & & & & & + & & + & & + & & & & & & & & & & & & & & & \\
\hline b1522 & Anger & & + & & & & & & & & & & & & & & & & & & & & & & & & & \\
\hline b1603 & Control of Thoughts & & & & & & & + & & & & & & & & & & & & & & & & & & & & \\
\hline b210 & Seeing function & & & & & & & & & & & & & & & & & & + & & & & & & & & & \\
\hline b255 & Smell function & & & & & & & & & & & & & & & & + & & & & & & & & & & & \\
\hline b265 & Touch sensation & & & & & & & & & & & & & & & & & + & & & & & & & & & & \\
\hline b280 & Sensation of Pain & & & & & & & & & & & & & & & & & & & & & & + & & & & & \\
\hline b440 & Respiratory functions & + & & & & & & & & & & & & & & & & & & & & & & & & & & \\
\hline b455 & $\begin{array}{l}\text { Exercise tolerance } \\
\text { functions }\end{array}$ & & & & & & & & & & & & & & & & & & & + & + & & + & + & & & & \\
\hline b4552 & Fatiguability & & & & & & & & & & & & + & + & & & & & & & & & + & & & & & \\
\hline b460 & $\begin{array}{l}\text { Sensation of } \\
\text { respiratory system }\end{array}$ & & & & & & & & & & & & & & & & + & & & & & & + & & & & & \\
\hline b730 & $\begin{array}{l}\text { Muscle power } \\
\text { functions }\end{array}$ & & & & & & & & & & & & & & + & + & & + & & & & & & & & & & \\
\hline
\end{tabular}


Table 3. Cont.

\begin{tabular}{|c|c|c|c|c|c|c|c|c|c|c|c|c|c|c|c|c|c|c|c|c|c|c|c|c|c|c|c|}
\hline & & & & & & & & & 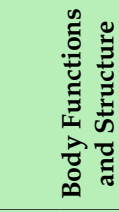 & & & & & & & & & & & 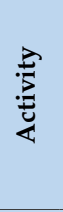 & & & 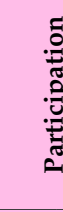 & & & 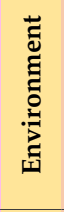 & 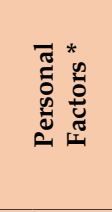 \\
\hline 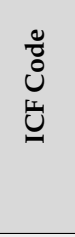 & 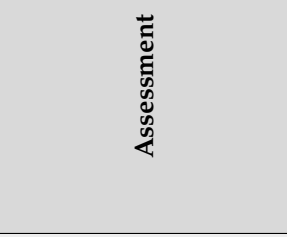 & 岳 & 芒 & 是 & 己్ & के & क्ष & $\mathrm{SCl} \underset{\stackrel{5}{\infty}}{\stackrel{2}{2}}$ & GH $\sum_{0}^{n}$ & $\underset{\substack{a \\
\underline{a}}}{a}$ & $\begin{array}{l}n \\
0 \\
n \\
n \\
\omega\end{array}$ & 品 & D & 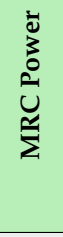 & 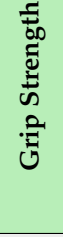 & है & 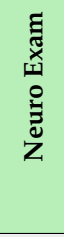 & 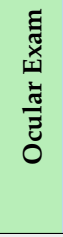 & $\sum_{0}^{\infty}$ & try & $\sum_{I}$ & 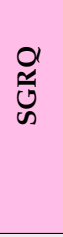 & $\underline{U}$ & की & $\begin{array}{l}\frac{\infty}{\infty} \\
\frac{1}{\infty}\end{array}$ & क्रे & 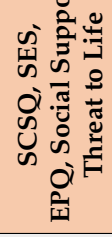 \\
\hline s220 & Structure of eyeball & & & & & & & & & & & & & & & & & + & & & & & & & & & \\
\hline d175 & Solving Problems & & & & & & & + & + & & & & & & & & & & & & + & & & & & & \\
\hline d177 & Making decisions & & & & & & & & + & & & & & & & & & & & & + & & & & & & \\
\hline d310 & $\begin{array}{l}\text { Communication with - } \\
\text { receiving - spoken } \\
\text { language }\end{array}$ & & & & & & & & & & & & & & & & & & & & + & & & & & & \\
\hline d330 & Speaking & & & & & & & & & & & & & & & & & & & & & & & & & & \\
\hline $\mathrm{d} 450$ & Walking & & & & & & & & & & & & & & & & & & + & & + & + & & & + & & \\
\hline d 460 & $\begin{array}{l}\text { Moving around in } \\
\text { different locations }\end{array}$ & & & & & & & & & & & & & & & & & & & & & + & & & + & & \\
\hline d510 & Washing oneself & & & & & & & & & & & & & & & & & & & & + & + & + & + & + & & \\
\hline d520 & Caring for body parts & & & & & & & & & & & & & & & & & & & & + & & & + & + & & \\
\hline d530 & Toileting & & & & & & & & & & & & & & & & & & & & + & & & + & & & \\
\hline d540 & Dressing & & & & & & & & & & & & & & & & & & & & + & + & & + & & & \\
\hline $\mathrm{d} 640$ & Doing housework & & & & & & & & & & & & & & & & & & & & & + & + & + & + & & \\
\hline $\mathrm{d} 710$ & $\begin{array}{l}\text { Basic interpersonal } \\
\text { interaction }\end{array}$ & & & & & & & & & & & & & & & & & & & & + & & & + & + & & \\
\hline d640 & Formal Relationship & & & & & & & & & & & & & & & & & & & & & & & + & & & \\
\hline d760 & Family Relationship & & & & & & & & & & & & & & & & & & & & & & & + & + & & \\
\hline $\mathrm{d} 770$ & Intimate Relationship & & & & & & & & & & & & & & & & & & & & & + & & + & & & \\
\hline d845 & $\begin{array}{l}\text { Work and } \\
\text { employment }\end{array}$ & & & & & & & & & & & & & & & & & & & & & + & & + & & & \\
\hline d920 & $\begin{array}{l}\text { Recreation and } \\
\text { Leisure }\end{array}$ & & & & & & & & & & & & & & & & & & & & & + & + & + & + & & \\
\hline e310 & Immediate family & & & & & & & & & & & & & & & & & & & & & & & & & + & \\
\hline e320 & Friends & & & & & & & & & & & & & & & & & & & & & & & & & + & \\
\hline
\end{tabular}




\section{Discussion}

Considering the widespread prevalence of COVID-19, the long-term complications of coronavirus infection in survivors will increase healthcare utilization significantly in the coming months. Previous outbreaks have shown that there is a broad spectrum of long-term complications of coronavirus infection which spans across all of the ICF components, with prevalence of respiratory compromise, exercise intolerance, psychological distress and reduced quality of life being considerably high several months following infection [5]. Deficits in different components do not necessarily translate into each other and there is no causal relationship between them [15]. The return of COVID-19 survivors to pre-infection levels of activity and participation in society are influenced not only by the degree of recovery from impairments in body structure and function but also by their personal and environmental factors, which hinder or facilitate their return to previous societal roles [54]. Therefore, it is essential that all future follow-up studies looking at COVID-19 survivors measure all aspects of the ICF framework. This review assimilates the outcome measures used in all the follow-up studies conducted during the present and past coronavirus outbreaks and classifies them according the ICF categories in order to provide a conceptual framework for the selection of outcome measures for future COVID-19 follow-up studies.

All studies included in this review reported outcomes in terms of body structure and function. The most commonly reported impairment in coronavirus survivors was respiratory compromise [9-12,34-36,39,42-44,48,55], with mainly restrictive patterns of lung function abnormality on pulmonary function testing. Neuromuscular impairment of muscle power and sensory function, particularly in those admitted to intensive care units, have also been reported based on neurological examination, MRC muscle power and grip strength measurements [13]. Long-term fatigue has also been measured using FSS and CFQ and found to be prevalent at 6 months post-discharge [24,27].

Alongside physical impairment, psychological impairments have also been widely elaborated by several studies. Prevalence of PTSD [23,24,26,27,29,38], depression [23,24,26,27] and anxiety [23,26] have been found to be particularly high in this cohort of patients. These mental health outcomes have been measured using a variety of scales. Outcome measures which were particularly useful in measuring impairment in these individuals were IES-R, PHQ-9 and GHQ-12 as these were able to capture the impact of psychological issues on a range of functions such as sleep, concentration, appetite and energy. We believe that at least two outcome measures should be used to assess impairment in body structure and function to address the physical and psychological impairment separately, such as a combination of lung function test and a PTSD outcome measure.

Limitations in activity have been measured through 6-min walking distance $[9,12,31,45]$ and cardiopulmonary testing in the included studies [34]. Most of the studies report these to be reduced following discharge, with gradual improvement at 6 months post-discharge. Participation has been measured using SF-36, SGRQ and SDSS. These reflect several domains of self-care, domestic life, interpersonal relationships, mobility, work and social life. Quality of life has been reported to be considerably reduced in coronavirus survivors $[11,29,31,42,56]$. Despite having extensive impact on the overall wellbeing of an individual, these tools have been measured by only a minority of studies and should be measured consistently across all future follow-up studies in COVID-19 survivors. We recommend at least one functional measure of activity, such as walking distance, and one to capture participation and quality of life, such as SF-36, for future studies.

Environmental factors have not been explored adequately by the included studies. Only one study measured these through the perceived social support scale [28]. None of the studies provided or reported information regarding pulmonary rehabilitation, pharmacological interventions or psychological support. As these interventions are also considered as environmental factors which could facilitate the recovery of these impairments in survivors, reporting of such factors in COVID-19 patients is also important. Attitudes of family and society members have also not been explored. Some of the measures which have been used to measure environmental factors in other areas of health have been the Craig Hospital Inventory of Environmental Factors (CHIEF), Environmental Factors Item Bank 
(EFIB), Facilitators and Barrier's Survey/Mobility (FABS/M) and Home and Community Environment Instrument (HACE). Future studies must aim to capture these factors along with family and carer support available in their chosen environments. Funding for rehabilitation will also play an important role in recovery for COVID-19 patients [57].

Personal factors which may play a role in recovery, such as coping styles, self-esteem, social stigma and personality, have been measured using self-constructed scales by some studies [24]. Some of the validated tools which have been used to measure such personal factors in other areas of health are the Connor Davidson resilience scale [58] and Kessler 6 psychological distress scale [59]. Inclusion of these measures could enable us to explore this domain further.

Through the use of the ICF framework, it is evident that, whilst impairments in body structure and function and restrictions in activity and participation have been measured extensively using standardized outcome measures, personal and environmental factors have only been measured in a small number of studies. The measurement of these contextual factors using standardized measures is essential as they have a major role to play in these individuals' health and return to function.

Figure 2 describes our ICF framework approach for selecting outcome measures for future studies looking at long-term outcomes after COVID-19 illness. Apart from the measures suggested in this framework, other outcomes which could be used to measure environmental and personal factors are the Connor Davidson resilience scale, Kessler 6 psychological distress scale, Craig Hospital Inventory of Environmental Factors (CHIEF), Environmental Factors Item Bank (EFIB), Facilitators and Barrier's Survey/Mobility (FABS/M) and Home and Community Environment Instrument (HACE) [60]. We propose that at least five different outcome measures spanning across all five ICF components need to be used in future follow-up studies.

It might be useful wherever possible for researchers to try to use the same outcome measures that were used in previous studies as this allows comparability and pooling of results. We however acknowledge that this might not be possible when there is a compelling case to use a measure that serves the purpose of the study better. For example, EQ5D is better suited to capture the health economics of the impact of the COVID-19 outbreak and researchers might opt to choose this over SF-36, which was predominantly used as the quality of life measure in previous studies.

The aim of this review was merely to categorize the currently available outcome measures into the ICF domains so that future researchers could pick an outcome measure corresponding to each category in order to capture the entire breadth of the health condition. We do not intend to make recommendations regarding specific outcome measures under each ICF domain that the reader should use. This would require further exploration of the psychometric properties of each measure, which is outside the scope of this paper. We only aim to provide a framework that one should keep in mind when choosing the measures rather than providing recommendations on which specific measure to use.

In the 36 studies included in this review, there were 33 different outcome measures used at follow-up. This makes comparison of results from studies using different measures difficult. In order to be able to do so, these diverse measures need to be converted into a common framework. This would most logically be done using the ICF, as it is the most commonly recognized international language of functioning. Thus, categorizing the measures into such a framework would be useful for researchers as it would identify the ICF categories that these authors found useful for their studies and inform their choice for future studies.

The main limitation of this review is that we have only described the outcome measures which have been used in the follow-up studies so far. This does not mean that outcome measures not used in these included studies are not suitable for use in future studies. Moreover, our search strategy was not designed to look for rehabilitation studies in coronavirus survivors. If these had been included in the review, then the set of outcome measures included in this review might have been slightly different. However, the aim of this review was to provide an ICF-based framework for the selection of outcome measures which researchers and clinicians are recommended to use. They need not necessarily use the same outcome measures as previous studies. For example, some researchers might prefer to use EQ5D 
instead of SF-36 for capturing quality of life. The rehabilitation community will be working hard in the next few years to help COVID-19 survivors achieve the best possible outcomes. The selection of outcome measures must be an essential first step rather than an afterthought in this process.

\section{Conclusions}

In conclusion, we are proposing an ICF-based framework to assist researchers in selecting outcome measures for future follow-up studies of COVID-19 survivors. This review highlighted that most studies so far placed greater emphasis on measuring body function impairments, limitations in activities and restrictions in participation. ICF personal and environmental factors were not as comprehensively covered and need to be included in COVID-19 follow-up studies. The individual ICF components are not linearly related and therefore a combination of outcome measures that capture all the components is recommended for a better understanding of the impact on survivors and planning interventions to maximize functional return.

Author Contributions: Conceptualization, K.P., M.S., N.Y.S., S.S., N.F. and J.L.M.; methodology, K.P. and M.S.; validation K.P., M.S., N.Y.S., S.S., N.F. and J.L.M.; formal analysis, K.P., M.S., N.Y.S., S.S., N.F. and J.L.M.; investigation, K.P. and M.S.; writing — original draft preparation, K.P.; writing-review and editing, K.P., M.S., N.Y.S., S.S., N.F. and J.L.M.; visualization, K.P.; supervision, M.S.; project administration, K.P. All authors have read and agreed to the published version of the manuscript.

Funding: This research received no external funding.

Conflicts of Interest: The authors declare no conflict of interest.

\section{References}

1. World Health Organization. Coronavirus Disease (COVID-19) Pandemic. 2020. Available online: https: //www.who.int/emergencies/diseases/novel-coronavirus-2019 (accessed on 31 May 2020).

2. Murray, A.; Gerada, C.; Morris, J. We Need a Nightingale Model for Rehab after COVID-19. Health Serv. J. 2020. Available online: https://www.hsj.co.uk/commissioning/we-need-a-nightingale-model-for-rehab-aftercovid-19-/7027335.article (accessed on 13 May 2020).

3. Carda, S.; Invernizzi, M.; Bavikatte, G.; Bensmaiil, D.; Bianchi, F.; Deltombe, T.; Draulans, N.; Esquenazi, A.; Francisco, G.E.; Gross, R.; et al. The role of physical and rehabilitation medicine in the COVID-19 pandemic: The clinician's view. Ann. Phys. Rehabil. Med. 2020. [CrossRef] [PubMed]

4. Holmes, E.A.; O'Connor, R.C.; Perry, V.H.; Tracey, I.; Wessely, S.; Arseneault, L.; Ballard, C.; Christensen, H.; Cohen Silver, R.; Everall, I.; et al. Multidisciplinary research priorities for the COVID-19 pandemic: A call for action for mental health science. Lancet Psychiatry 2020. [CrossRef]

5. Ahmed, H.; Patel, K.; Greenwood, D.; Halpin, S.; Lewthwaite, P.; Salawu, A.; Eyre, L.; Breen, A.; O'Connor, R.; Jones, A.; et al. Long-term clinical outcomes in survivors of severe acute respiratory syndrome and Middle East respiratory syndrome coronavirus outbreaks after hospitalisation or ICU admission: A systematic review and meta-analysis. J. Rehabil. Med. 2020. [CrossRef] [PubMed]

6. Chan, K.S.; Zheng, J.P.; Mok, Y.W.; Li, Y.M.; Liu, Y.N.; Chu, C.M.; Ip, M.S. SARS: Prognosis, outcome and sequelae. Respirology 2003, 8, S36-S40. [CrossRef] [PubMed]

7. Carfi, A.; Bernabei, R.; Landi, F. Persistent Symptoms in Patients After Acute COVID-19. JAMA 2020. [CrossRef]

8. Halpin, S.J.; McIvor, C.; Whyatt, G.; Adams, A.; Harvey, O.; McLean, L.; Walshaw, C.; Kemp, S.; Corrado, J.; Singh, R.; et al. Postdischarge symptoms and rehabilitation needs in survivors of COVID-19 infection: A cross-sectional evaluation. J. Med. Virol. 2020. [CrossRef]

9. Hui, D.S.; Wong, K.T.; Ko, F.W.; Tam, L.S.; Chan, D.P.; Woo, J.; Sung, J.J.Y. The 1-year impact of severe acute respiratory syndrome on pulmonary function, exercise capacity, and quality of life in a cohort of survivors. Chest 2005, 128, 2247-2261. [CrossRef]

10. Chen, J.H.; Ma, D.Q.; He, W.; Jin, E.H.; Zhang, J.; Zhong, Z.H. Follow-up study of chest CT manifestations of patients with severe acute respiratory syndrome. Chin. J. Radiol. 2006, 40, 1161-1165. 
11. Tansey, C.M.; Louie, M.; Loeb, M.; Gold, W.L.; Muller, M.P.; De Jager, J.A.; Cameron, J.I.; Tomlinson, G.; Mazzulli, T.; Walmsley, S.L.; et al. One-year outcomes and health care utilization in survivors of severe acute respiratory syndrome. Arch. Intern. Med. 2007, 167, 1312-1320. [CrossRef]

12. Park, W.B.; Jun, K.I.; Kim, G.; Choi, J.-P.; Rhee, J.-Y.; Cheon, S.; Lee, C.H.; Park, J.-S.; Kim, Y.; Joh, J.-S.; et al. Correlation between Pneumonia Severity and Pulmonary Complications in Middle East Respiratory Syndrome. J. Korean Med. Sci. 2018, 33, e169. [CrossRef]

13. Tsai, L.K.; Hsieh, S.T.; Chao, C.C.; Chen, Y.C.; Lin, Y.H.; Chang, S.C.; Chang, Y.C. Neuromuscular disorders in severe acute respiratory syndrome. Arch. Neurol. 2004, 61, 1669-1673. [CrossRef] [PubMed]

14. Sivan, M.; Sell, B.; Sell, P. A comparison of functional assessment instruments and work status in chronic back pain. Eur. J. Phys. Rehabil. Med. 2009, 45, 31-36.

15. Müller, U.; Roeder, C.; Dubs, L.; Duetz, M.S.; Greenough, C.G. Condition-specific outcome measures for low back pain. Eur. Spine J. 2004, 13, 314-324. [CrossRef] [PubMed]

16. Stucki, G.; Ewert, T.; Cieza, A. Value and application of the ICF in rehabilitation medicine. Disabil. Rehabil. 2003, 25, 628-634. [CrossRef]

17. World Health Organization. World Health Organization International Classification of Functioning, Disability and Health; World Health Organization: Geneva, Switzerland, 2001.

18. Peng, M.; Cai, B.; Liu, T.; Ma, Y.; Xu, W.; Cao, B.; Shi, J.; Han, J.; Zhang, W. Assessment of pulmonary function in SARS patients during the convalescent period. Zhongguo Yi Xuе Ke Xue Yuan Xue Bao. 2003, 25, 529-532. [PubMed]

19. Sivan, M.; O'Connor, R.J.; Makower, S.; Levesley, M.; Bhakta, B. Systematic review of outcome measures used in the evaluation of robot-assisted upper limb exercise in stroke. J. Rehabil. Med. 2011, 43, 181-189. [CrossRef]

20. Bilbao, A.; Kennedy, C.; Chatterji, S.; Üstün, B.; Vásquez Barquero, J.L.; Barth, J.T. The ICF: Applications of the WHO model of functioning, disability and health to brain injury rehabilitation. NeuroRehabilitation 2003, 18, 239-250. [CrossRef]

21. Peeri, N.C.; Shrestha, N.; Rahman, M.S.; Zaki, R.; Tan, Z.; Bibi, S.; Baghbanzadeh, M.; Aghamohammadi, N.; Zhang, W.; Haque, U. The SARS, MERS and novel coronavirus (COVID-19) epidemics, the newest and biggest global health threats: What lessons have we learned? Int. J. Epidemiol. 2020, 49, 717-726. [CrossRef]

22. Cieza, A.; Geyh, S.; Chatterji, S.; Kostanjsek, N.; Üstün, B.; Stucki, G. ICF linking rules: An update based on lessons learned. J. Rehabil. Med. 2005, 37, 212-218. [CrossRef]

23. Mak, I.W.C.; Chu, C.M.; Pan, P.C.; Yiu, M.G.C.; Chan, V.L. Long-term psychiatric morbidities among SARS survivors. Gen. Hosp. Psychiatry 2009, 31, 318-326. [CrossRef]

24. Lam, M.; Wing, Y.; Yu, M.; Leung, C.; Ma, R.; Kong, A.; So, W.; Fong, S.; Lam, S. Mental morbidities and chronic fatigue in severe acute respiratory syndrome survivors: Long-term follow-up. Arch. Intern. Med. 2009, 169, 2142-2147. [CrossRef] [PubMed]

25. Wu, K.K.; Chan, S.K.; Ma, T.M. Posttraumatic stress, anxiety, and depression in survivors of severe acute respiratory syndrome (SARS). J. Trauma. Stress 2005, 18, 39-42. [CrossRef] [PubMed]

26. Lee, A.M.; Wong, J.G.W.S.; McAlonan, G.M.; Cheung, V.; Cheung, C.; Sham, P.C.; Chu, C.-M.; Wong, P.-C.; Tsang, K.W.T.; Chua, S.E. Stress and psychological distress among SARS survivors 1 year after the outbreak. Can. J. Psychiatry. 2007, 52, 233-240. [CrossRef]

27. Lee, S.H.; Shin, H.S.; Park, H.Y.; Kim, J.L.; Lee, J.J.; Lee, H.; Won, S.D.; Han, W. Depression as a mediator of chronic fatigue and post-traumatic stress symptoms in middle east respiratory syndrome survivors. Psychiatry Investig. 2019, 16, 59-64. [CrossRef] [PubMed]

28. Zhang, K.; Xu, Y.; Liu, Z.; Yang, H.; Song, L.; Xue, Y.; Lu, L.; Peng, C.; ZQ, C.; Wu, H.; et al. Controlled study of posttraumatic stress disorder among patients with severe acute respiratory syndrome and first-line hospital staffs as well as public in prevalent areas. Chin. J. Clin. Rehabil. 2005, 9, 94-96.

29. Hong, X.; Currier, G.W.; Zhao, X.; Jiang, Y.; Zhou, W.; Wei, J. Posttraumatic stress disorder in convalescent severe acute respiratory syndrome patients: A 4-year follow-up study. Gen. Hosp. Psychiatry 2009, 31, 546-554. [CrossRef]

30. Liu, K.; Zhang, W.; Yang, Y.; Zhang, J.; Li, Y.; Chen, Y. Respiratory rehabilitation in elderly patients with COVID-19: A randomized controlled study. Complement. Ther. Clin. Pract. 2020, 39, 101166. [CrossRef] 
31. Lam, S.P.; Tsui, E.; Chan, K.S.; Lam, C.L.; So, H.P. The validity and reliability of the functional impairment checklist (FIC) in the evaluation of functional consequences of severe acute respiratory distress syndrome (SARS). Qual. Life Res. 2006, 15, 217-231. [CrossRef]

32. Xie, L.; Liu, Y.; Fan, B.; Xiao, Y.; Tian, Q.; Chen, L.; Zhao, H.; Chen, W. Dynamic changes of serum SARS-Coronavirus IgG, pulmonary function and radiography in patients recovering from SARS after hospital discharge. Respir. Res. 2005, 6, 5. [CrossRef]

33. He, Z.Y. A follow-up study of the lung function and the chest CT changes in medical staff with severe acute respiratory syndrome in Beijing. Chinese J. Tuberc. Respir. Dis. 2005, 28, 10-12.

34. Ong, K.; Ng, A.; Lee, L.; Kaw, G.; Kwek, S.; Leow, M.; Earnest, A. Pulmonary function and exercise capacity in survivors of severe acute respiratory syndrome. Eur. Respir. J. 2004, 24, 436-442. [CrossRef] [PubMed]

35. Ng, C.K.; Chan, J.W.M.; Kwan, T.L.; To, T.S.; Chan, Y.H.; Ng, F.Y.Y.; Mok, T.Y.W. Six month radiological and physiological outcomes in severe acute respiratory syndrome (SARS) survivors. Thorax 2004, 59, 889-891. [CrossRef] [PubMed]

36. Zheng, Z.; Chen, R.; Wu, H.; Liu, X.; He, W.; Xu, Y.; Chen, S.; Li, Y.; Zheng, J.; Zhong, N. Changes in pulmonary function in severe acute respiratory syndrome patients during convalescent period. Chin. Crit. Care Med. 2005, 17, 329-331. [CrossRef]

37. Hsu, H.; Tzao, C.; Wu, C.; Chang, W.; Tsai, C.; Tung, H.; Chen, C. Correlation of high-resolution CT, symptoms, and pulmonary function in patients during recovery from severe acute respiratory syndrome. Chest 2004, 126, 149-158. [CrossRef]

38. Gao, H.; Hui, W.; Lan, X. A follow-up study of post-traumatic stress disorder of SARS patients after discharge. Chin. J. Rehabil. Med. 2006, 21, 3-5.

39. Zheng-yu, J.; Hui, Y.; Wei-hong, Z.; Yun, W.; Ji-Xiang, L.; Wen-bin, M.; Min, P.; Yi, M.; Bai-qiang, C.; Zhong, W.; et al. Thoracic high resolution CT findings of 100 SARS patients in convalescent period. Acta Acad. Med. Sin. 2003, 25, 512-515.

40. Han, Y.; Geng, H.; Feng, W.; Tang, X.; Ou, A.; Lao, Y.; Xu, Y.; Lin, H.; Liu, H.; Li, Y.; et al. A follow-up study of 69 discharged SARS patients. J. Tradit. Chinese Med. 2003, 23, 214-217.

41. Liu, Y.; Ye, Y.; Zhang, P.; Chen, J.; Ye, H.; He, Y.; Li, N. Changes in pulmonary function in SARS patients during the three-year convalescent period. Zhongguo Wei Zhong Bing Ji Jiu Yi Xue 2007, 19, 536-538.

42. Ong, K.; Ng, A.; Lee, L.; Kaw, G.; Kwek, S.; Leow, M.; Earnest, A. 1-year pulmonary function and health status in survivors of severe acute respiratory syndrome. Chest 2005, 128, 1393-1400. [CrossRef]

43. Zhang, P.; Li, J.; Liu, H.; Han, N.; Ju, J.; Kou, Y.; Chen, L.; Jiang, M.; Pan, F.; Zheng, Y.; et al. Long-term consequences in lung and bone associated with hospital-acquired severe acute respiratory syndrome: A 15-year follow-up from a prospective cohort study. Lancet 2020, 8, S11. [CrossRef]

44. Wong, K.; Antonio, G.E.; Hui, D.S.C.; Ho, C.; Chan, P.; Ng, W.; Shing, K.; Wu, A.; Lee, N.; Yap, F.; et al. Severe Acute Respiratory Syndrome Thin-Section Computed Tomography Features, Temporal Changes, and Clinicoradiologic Correlation During the Convalescent Period. Lung 2004, 28, 790-795.

45. Li, T.S.; Gomersall, C.D.; Joynt, G.M.; Chan, D.P.S.S.; Leung, P.; Hui, D.S.C.C. Long-term outcome of acute respiratory distress syndrome caused by severe acute respiratory syndrome (SARS): An observational study. Crit. Care Resusc. 2006, 8, 302-308.

46. Chiang, C.H.; Shih, J.F.; Su, W.J.; Perng, R.P. Eight-month prospective study of 14 patients with hospital-acquired severe acute respiratory syndrome. Mayo Clin. Proc. 2004, 79, 1372-1379. [CrossRef] [PubMed]

47. Yin, C.; Wang, C.; Wen, Y.; Jiang, L.; Lu, Q.; Li, J.; Wang, J.; HE, Z. Prospective 2-year clinical study of patients with positive IgG-antibodies after recovering from severe acute respiratory syndrome. Chin. Crit. Care Med. 2005, 17, 740-742.

48. Wu, X.; Dong, D.; Ma, D. Thin-section computed tomography manifestations during convalescence and long- term follow-up of patients with severe acute respiratory syndrome (SARS). Med. Sci. Monit. 2016, 22, 2793-2799. [CrossRef] [PubMed]

49. Avendano, M.; Derkach, P.; Swan, S. Clinical course and management of SARS in health care workers in Toronto: A case series. CMAJ 2003, 168, 1649-1660. [PubMed]

50. Isakbaeva, E.; Khetsuriani, N.; Beard, R.; Peck, S.; Erdman, D.; Monroe, S.; Tong, S.; Ksiazek, T.; Lowther, S.; Pandya-Smith, I.; et al. SARS-associated Coronavirus Traansmission, United States. Emerg. Infect. Dis. 2004, 10, 225-231. [CrossRef] 
51. Klopfenstein, T.; Kadiane-Oussou, N.; Toko, L.; Royer, P.; Lepiller, Q.; Gendrin, V.; Zayet, S. Features of anosmia in COVID-19. Médecine Mal. Infect. 2020, 50, 436-439. [CrossRef]

52. Hopkins, C.; Surda, P.; Whitehead, E.; Kumar, B.N. Early recovery following new onset anosmia during the COVID-19 outbreak-An observational cohort study. J. Otolaryngol.-Head Neck Surgery 2020, 49, 1-6. [CrossRef]

53. Yuen, K.S.C.; Chan, W.M.; Fan, D.S.P.; Chong, K.K.L.; Sung, J.J.Y.; Lam, D.S.C. Ocular screening in severe acute respiratory syndrome. Am. J. Ophthalmol. 2004, 137, 773-774. [CrossRef]

54. Functioning and Disability Reference Group The ICF: An Overview. World Health Organ. 2010. Available online: https://www.cdc.gov/nchs/data/icd/icfoverview_finalforwho10sept.pdf (accessed on 3 September 2020).

55. Xie, L.; Liu, Y.; Xiao, Y.; Tian, Q.; Fan, B.; Zhao, H.; Chen, W. Follow-up study on pulmonary function and lung radiographic changes in rehabilitating severe acute respiratory syndrome patients after discharge. Chest 2005, 127, 2119-2124. [CrossRef] [PubMed]

56. Hui, D.; Wong, K.; Antonio, G.; Tong, M.; Chan, D.; Sung, J. Long-term sequelae of SARS: Physical, neuropsychiatric, and quality-of-life. Hong Kong Med. J. 2009, 15, 21-23. [PubMed]

57. Xu, X.; Zhang, L.; Chen, L.; Wei, F. Does COVID-2019 have an Impact on the Purchase Intention of Commercial Long-Term Care Insurance among the Elderly in China? Healthcare 2020, 8, 126. [CrossRef] [PubMed]

58. Ehrich, J.; Mornane, A.; Powern, T. Psychometric Validation of the 10-item Connor-Davidson Resilience Scale. J. Appl. Meas. 2017, 18, 122-136.

59. Kang, Y.K.; Guo, W.J.; Xu, H.; Chen, Y.H.; Li, X.J.; Tan, Z.P.; Li, N.; Gesang, Z.R.; Wang, Y.M.; Liu, C.B.; et al. The 6-item Kessler psychological distress scale to survey serious mental illness among Chinese undergraduates: Psychometric properties and prevalence estimate. Compr. Psychiatry 2015, 63, 105-112. [CrossRef] [PubMed]

60. Heinemann, A.W.; Miskovic, A.; Semik, P.; Wong, A.; Dashner, J.; Baum, C.; Magasi, S.; Hammel, J.; Tulsky, D.S.; Garcia, S.F.; et al. Measuring Environmental Factors: Unique and Overlapping International Classification of Functioning, Disability and Health Coverage of 5 Instruments Archives of Physical Medicine and Rehabilitation. Arch. Phys. Med. Rehabil. 2016, 97, 2113-2135. [CrossRef]

(C) 2020 by the authors. Licensee MDPI, Basel, Switzerland. This article is an open access article distributed under the terms and conditions of the Creative Commons Attribution (CC BY) license (http://creativecommons.org/licenses/by/4.0/). 